Supporting Information

\title{
Plasmonic Hot Electron-Mediated Hydrodehalogenation Kinetics on Nanostructured Ag Electrodes
}

Jia Liu, Zhuan-Yun Cai, Wei-Xin Sun, Jia-Zheng Wang, Xiao-Ru Shen, Chao Zhan, Rajkumar Devasenathipathy, Jian-Zhang Zhou*, De-Yin Wu*, Bing-Wei Mao and Zhong-Qun Tian

State Key Laboratory of Physical Chemistry of Solid Surfaces and Department of Chemistry, College of Chemistry and Chemical Engineering, Xiamen University, Xiamen 361005, China

*Corresponding authors, E-mail: dywu@xmu.edu.cn; jzzhou@xmu.edu.cn.

\section{This PDF file includes:}

1. Experimental section.

2. The derivation of the reaction mechanism for observed rate constant $\boldsymbol{k}_{\boldsymbol{\theta}}$.

3. Electrochemical cyclic voltammetry of 8BrAd.

4. EC-SERS of 8BrAd in different $\mathrm{pH}$ values.

5. CV curves of photoelectrochemical cell

6. Calculation details of electron transfer rate constants and the energy of molecules.

7. The derivation of $\boldsymbol{k}_{\boldsymbol{l}}$ by absorption coefficient $\boldsymbol{\alpha}$.

8. Finite Element Method (FEM) simulation of absorption of plasmonic Ag electrode of nanostructure.. 


\section{Experimental section}

\section{Preparation of Ag NPs@Ag electrode.}

Silver nanoparticles (Ag NPs) were prepared following the typical citrate reduction method proposed by Lee and Meisel ${ }^{1}$. The colloids were centrifuged and washed twice to remove the protected citrate for using. The silver electrode was polished to a mirror finish with emery paper of decreasing grain size followed by alumina powder with 1 and $0.05 \mu \mathrm{m}$ particle sizes in this sequence. It was cleaned by sonication in Millipore water for 3 min after each polishing step. The Ag NPs were dropped on the mechanically polished Ag electrode to achieve a self-assembled SERS active Ag NPs modified electrode $^{2}$. The modified electrode was immersed into the ethanol solution of $0.3 \mathrm{mM} 8$ $\mathrm{Br}$ adenine or $0.3 \mathrm{mM}$ adenine for $3 \mathrm{~h}$, after that, the physically adsorbed molecules were washed away by copious of Millipore water.

Scanning electron microscopy (SEM) images were recorded with a HITACHI S-4800. The extinction spectra and the scan electron microscopy (SEM) imaging showing produced Ag NPs were about $70 \pm 10 \mathrm{~nm}$ in diameter. The UV-Vis absorption spectra of Ag sol-gel were recorded on a Shimadzu UV-2100 spectrometer. The UV-Vis reflectance spectra were obtained in a homemade device.
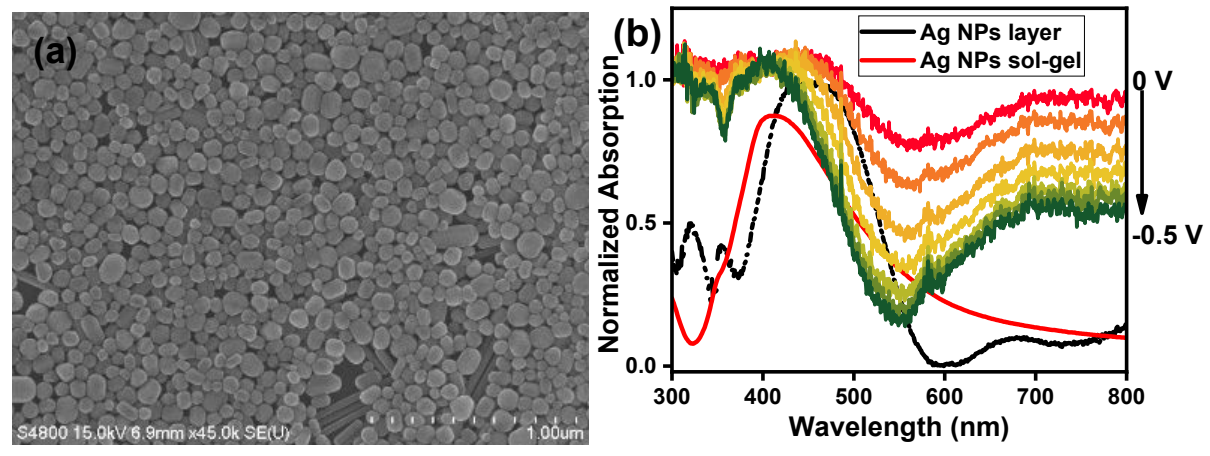

Figure S1. (a) Scanning electron microscopy (SEM) of Ag NPs (b) the UV-Vis absorption spectra and the UV-Vis electro-reflectance spectra of the Ag NPs@Ag electrode in homemade reflectance spectrometer. The potential step of electroreflectance spectra were collected from $0 \mathrm{~V}$ to $-0.5 \mathrm{~V}$ vs. $\mathrm{Ag} \mid \mathrm{AgCl}$, the potential interval is $0.1 \mathrm{~V}$. 


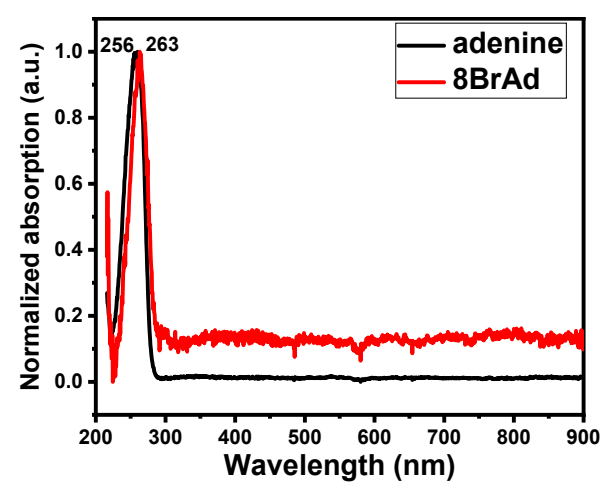

Figure S2. The UV-Visible absorption spectra of $8 \mathrm{BrAd}$ and adenine of $0.3 \mathrm{mM}$ aqueous solution.

\section{Electrochemical and spectroelectrochemical measurements.}

Electrochemical experiments were carried out using a CHI660e electrochemical workstation (CH Instruments, China). Silver electrode (2 mm diameter) covered with Ag nanoparticles act as a working electrode. The in-situ electrochemical cell consisted of a Ag NPs@Ag working electrode, a platinum wire counter electrode and an Ag|AgCl reference electrode.

SERS experiments were performed on a WITec confocal Raman microscope. SERS signals were excited by laser $532 \mathrm{~nm}$ or $633 \mathrm{~nm}$ with a power of $0.1 \mathrm{~mW}$ on samples and collected using a 50X objective with an $\mathrm{N}_{\mathrm{A}}$ of 0.55 . SERS signals excited by $785 \mathrm{~nm}$ laser were conducted on the confocal Raman system Xplora (Jobin-Yvon Horiba). The acquisition time was $0.5 \mathrm{~s}$ for the time series SERS spectra. The potential of the electrode was controlled by a potentiostat (PGSTAT 101, Autolab).

\section{Chemical reagents.}

All reagents employed were commercially available and used without further purification. 8BrAd was purchased from Alfa Aesar. Adenine and deuterated water was obtained from Sigma-Aldrich. Silver nitrate was obtained from Sinopharm Chemical Reagent Co. Deionized ultrapure water $(18.2 \mathrm{M} \Omega \cdot \mathrm{cm})$ from a Millipure Milli-Q system was used throughout this study.

\section{The derivation of the reaction mechanism for observed rate constant $k_{0}$.}

PMCR was realized by the SERS measurement of $8 \mathrm{BrAd}$ adsorbed on the AgNPs @ Ag electrode in $0.2 \mathrm{M}$ phosphate buffer solution. There is no molecule $8 \mathrm{BrAd}$ in the electrolyte and diffusion can be neglected. The rate constant $k_{0}$ focuses on the surface adsorbed $8 \mathrm{BrAd}$ reaction: 


$$
\begin{gathered}
\mathrm{M} \underset{\mathrm{k}_{1}}{\stackrel{\mathrm{hu}}{\longrightarrow}} \mathrm{M}^{*} \\
8 \mathrm{BrAd}+\mathrm{e}^{-}\left(\mathrm{M}^{*}\right) \stackrel{\mathrm{k}_{2}}{\underset{\mathrm{k}_{-2}}{\longrightarrow}} 8 \mathrm{BrAd}^{\bullet-}(+\mathrm{M}) \\
8 \mathrm{BrAd}^{\bullet-} \stackrel{\mathrm{k}_{3}}{\longrightarrow} \mathrm{Ad}^{\bullet}+\mathrm{Br}^{-}
\end{gathered}
$$

The reaction rate of the reactant $8 \mathrm{BrAd}$ could be expressed as:

$$
\frac{d[8 B r A d]}{d t}=-k_{2}[M *][8 B r A d]+k_{-2}[M]\left[8 B r A d^{g-}\right]
$$

According to the steady-state approximation, the reaction rate of the intermediate transient negative ion could be describe as:

$$
\begin{gathered}
\frac{d\left[8 B r A d^{g-}\right]}{d t}=0 \\
\frac{d\left[8 B r A d^{g-}\right]}{d t}=k_{2}[M *][8 B r A d]-k_{-2}[M]\left[8 B r A d^{g-}\right]-k_{3}\left[8 B r A d^{g-}\right]=0 \\
{\left[8 B r A d^{g-}\right]=\frac{k_{2}[M *][8 B r A d]}{k_{-2}[M]+k_{3}}}
\end{gathered}
$$

The equation (8) could be assumed from the equations (4) and (7) to determine the observed rate constant $k_{o}$ of the dissociation of $8 \mathrm{BrAd}$ :

$$
\ln \frac{[8 B r A d] t}{[8 B r A d]_{0}}=k_{o}, k_{o}=\frac{-k_{2} k_{3}[M *]}{k_{-2}[M]+k_{3}}
$$

Hence pseudo first-order kinetics is assumed to determine the observed rate constant $k_{o}=\frac{1}{t} \ln \frac{[8 B r A d]_{t}}{[8 B r A d] 0}, \mathrm{M}^{*}$ is relevant to the hot electrons generated from the excited surface plasmon induced by light. [ $\left.\mathrm{M}^{*}\right]$ could be described as $\mathrm{k}_{1}[\mathrm{M}]$. The $k_{o}$ of different potentials could be detected by time dependent spectra. And the reaction rate constant could be written as: 


$$
\frac{1}{k_{0}}=\frac{k_{-2}}{k_{1} k_{2}}\left(\frac{1}{k_{-2}[M]}+\frac{1}{k_{3}}\right)
$$

If the cleavage of transient negative ion is very fast, which means $\mathrm{k}_{3}>>\mathrm{k}_{-2}\left[\mathrm{M}^{+}\right]$, the reaction rate constant could be determined as $\mathrm{k}_{0}=\mathrm{k}_{1} \mathrm{k}_{2}[\mathrm{M}]$, the apparent rate constant of the reaction is determined by the generation of hot electrons and electron transfer to 8 BrAd molecules adsorbed on surfaces. This was influenced the surface plasmon resonance effect of AgNPs modified silver electrode, incident light intensity, and wavelengths.

If the $\mathrm{k}_{-2}[\mathrm{M}]>>\mathrm{k}_{3}, k_{o}=\frac{k_{1} k_{2} k_{3}}{k_{-2}}$, and could be written as

$$
k_{o}=K k_{1} k_{3}, K=k_{2} / k_{-2},
$$

where $\mathbf{K}$ was assumed in relation to a given potential. The whole reaction was treated as a single step as the electrocatalytic dissociation of aromatic bromides. The changes in $\boldsymbol{k}_{\boldsymbol{o}}$ indicates the synergistic effect between light and the applied potentials in the PMCR.

For the formation of adenine, we propose the following processes,

$$
\begin{gathered}
\mathrm{Ad} \cdot+\mathrm{e}^{-} \underset{\mathrm{k}_{-4}}{\stackrel{\mathrm{k}_{4}}{\longrightarrow}} \mathrm{Ad}^{-} \\
\mathrm{Ad}^{-}+\mathrm{SH} \stackrel{\mathrm{k}_{5}}{\longrightarrow} \mathrm{Ad}+\mathrm{S}^{-} \\
\mathrm{Ad}^{\bullet}+\mathrm{SH} \stackrel{\mathrm{k}_{6}}{\longrightarrow} \mathrm{Ad}+\mathrm{S}^{\cdot} \\
\mathrm{Ad}^{\cdot}+8 \mathrm{BrAd}^{-} \stackrel{\mathrm{k}_{6}}{\longrightarrow} \mathrm{Ad}^{-}+8 \mathrm{BrAd}
\end{gathered}
$$

The reaction rate of adenine formation could be represented as follows,

$$
\frac{d[A d]}{d t}=k_{5}\left[A d^{-}\right]\left[H^{+}\right]+k_{6}[A d \mathrm{~g}][H S]
$$

According to the steady-state approximation, the intermediate reaction rates of the radical and negative ion could be described as, 


$$
\begin{gathered}
\frac{d[A d \mathrm{~g}]}{d t}=k_{3}\left[8 B r A d^{g-}\right]-k_{4}[A d \mathrm{~g}]+k_{-4}\left[A d^{-}\right]-k_{6}[A d \mathrm{~g}][H S]=0 \\
\frac{d\left[A d^{-}\right]}{d t}=k_{4}[A d \mathrm{~g}]-k_{-4}\left[A d^{-}\right]-k_{5}\left[A d^{-}\right]\left[H^{+}\right]=0
\end{gathered}
$$

From equations (13) and (14), the increase of product adenine could be written as,

$$
\begin{gathered}
\frac{d[A d]}{d t}=k_{3}\left[8 B r A d^{g-}\right]=\frac{k_{3} k_{2}[M *][8 B r A d]}{k_{-2}[M]+k_{3}} \\
{[A d]=[8 B r A d]_{0}-[8 B r A d]_{0} \exp \left(k_{p} t\right)} \\
\text { and } k_{p}=-k_{0}=\frac{k_{3} k_{2}[M *]}{k_{-2}[M]+k_{3}}
\end{gathered}
$$

The SERS intensity of the observed band is assumed to be proporational to the Raman scattering cross-section. ${ }^{3}$ Now we can obtain reactant $8 \mathrm{BrAd}$ and product Ad on the surface,

$$
\begin{gathered}
I_{731}=I N_{0} \sigma_{A d}[A d]+\text { baseline } \\
\text { and } \\
I_{763}=I N_{0} \sigma_{8 B r d d}[8 B r A d]+\text { baseline }
\end{gathered}
$$

Here $\sigma_{8 \mathrm{brAd}}$ and $\sigma_{\mathrm{Ad}}$ are the Raman cross sections of $8 \mathrm{BrAd}$ and Ad, respectively. $N_{0}$ is Avogadro constant, $I_{731}$ and $I_{763}$ are the intensity of the ring breathing vibration in adenine and $8 \mathrm{BrAd}$, and represents the change of surface concentrations[Ad] and [8BrAd] with time. It was noted that the baseline remains constants at same potentials. So we adopted the baseline as zero in the fitting procedures. Thus, the $\boldsymbol{k}_{\boldsymbol{p}}$ and $\boldsymbol{k}_{\boldsymbol{\theta}}$ in $\mathrm{pH} 8$ were caculated to make a comparision.

Table S2-1. The rate constant $k_{0}$ at different potentials fitted from the intensity profiles by using $\mathrm{I}_{763}-\mathrm{I}_{\text {baseline }}=\mathrm{A} * \exp \left(-k_{0} \mathrm{t}\right)$ at $532 \mathrm{~nm}$ laser with a power $0.05 \mathrm{~mW}$ 


\begin{tabular}{ccccccccc}
\hline Potential/V & $\mathbf{0}$ & $\mathbf{- 0 . 0 5}$ & $\mathbf{- 0 . 1}$ & $\mathbf{- 0 . 1 5}$ & $\mathbf{- 0 . 2}$ & $\mathbf{- 0 . 2 5}$ & $\mathbf{- 0 . 3}$ & $\mathbf{- 0 . 3 5}$ \\
& & & & & & & & \\
\hline \multirow{2}{*}{$\boldsymbol{k}_{0}$} & 0.0088 & 0.00819 & 0.01417 & 0.02073 & 0.02943 & 0.03997 & 0.04704 & 0.07504 \\
& & \pm & \pm & \pm & \pm & \pm & \pm & \pm \\
& \pm & 0.00850 & 0.00178 & 0.00198 & 0.00296 & 0.000922 & 0.00148 & 0.00795
\end{tabular}

Table S2-2 The rate constant $k_{0}$ at different potentials fitted from the intensity profiles by using $\mathrm{I}_{731}-\mathrm{I}_{\text {baseline }}=\mathrm{A}^{*} \exp \left(\mathrm{k}_{p} \mathrm{t}\right)$ at $532 \mathrm{~nm}$ laser

\begin{tabular}{ccccccccc}
\hline Potential/V & $\mathbf{0}$ & $\mathbf{- 0 . 0 5}$ & $\mathbf{- 0 . 1}$ & $\mathbf{- 0 . 1 5}$ & $\mathbf{- 0 . 2}$ & $\mathbf{- 0 . 2 5}$ & $\mathbf{- 0 . 3}$ & $\mathbf{- 0 . 3 5}$ \\
& & & & & & & & \\
\hline \multirow{2}{*}{$\boldsymbol{k}_{p}$} & 0.01287 & 0.01199 & 0.01666 & 0.01780 & 0.02651 & 0.03636 & 0.04477 & 0.05567 \\
& \pm & \pm & \pm & \pm & \pm & \pm & \pm & \pm \\
& 0.000595 & 0.00577 & 0.00326 & 0.00356 & 0.0019 & 0.00291 & 0.00122 & \\
& & & & & & & & 0.0115 \\
\hline
\end{tabular}

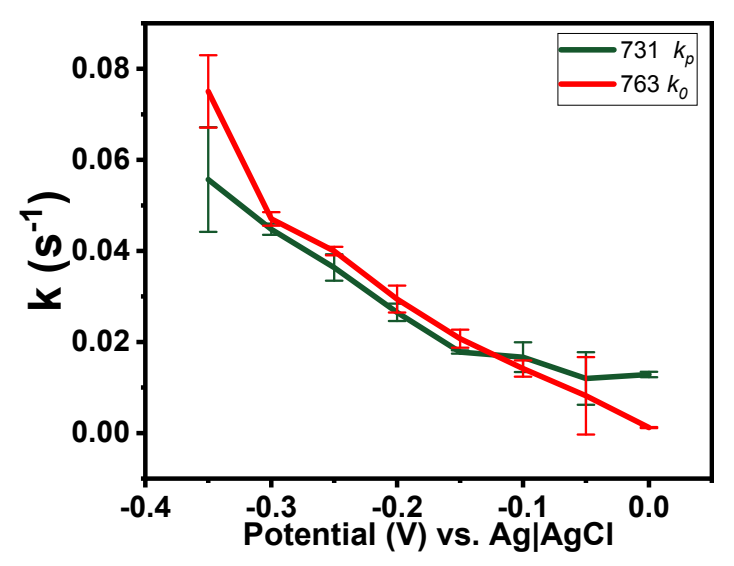

Figure S3. The variation of rate constants $\boldsymbol{k}_{\boldsymbol{0}}$ and $\boldsymbol{k}_{\boldsymbol{p}}$ with applied potentials. The PMCR was probed by SERS intensities in $532 \mathrm{~nm}$, Laser power is $0.05 \mathrm{~mW}$, pH of 8 in PBS solutions. The $\boldsymbol{k}_{\boldsymbol{0}}$ and $\boldsymbol{k}_{\boldsymbol{p}}$ could be described as $k_{0}=a k_{p}, a=\ln \sigma_{s B r t a} / \sigma_{A d}$, and a $\approx 0.925, \boldsymbol{k}_{\boldsymbol{0}} \approx$ $\boldsymbol{k}_{\boldsymbol{p}}$. The $\boldsymbol{k}_{\boldsymbol{p}}$ was shown as the observed rate constant in this paper. 
According to the $5 / 2$ power law, a linear relationship of photocurrent $\underline{i}_{\underline{p}}$ is fitted with electrode potential at the same laser power. The photocurrent $i_{p}$ is written as,

$i_{p}=n F k_{0}[8 B r A d] e^{-k t}$, At the beginning of the reaction, the photocurrent could be written as $i_{p}=n F k_{0}[8 B r A d]_{0}$, and the $i_{p}$ is proportional to the rate constant. This observation indicates that the $i_{p} \propto \boldsymbol{k}_{\boldsymbol{0}}$.

\section{Electrochemical cyclic voltammetry of $8 B$ Bd.}

Influence of scanning rates on cycle voltammogram (CV) curves is shown in Figure S4.
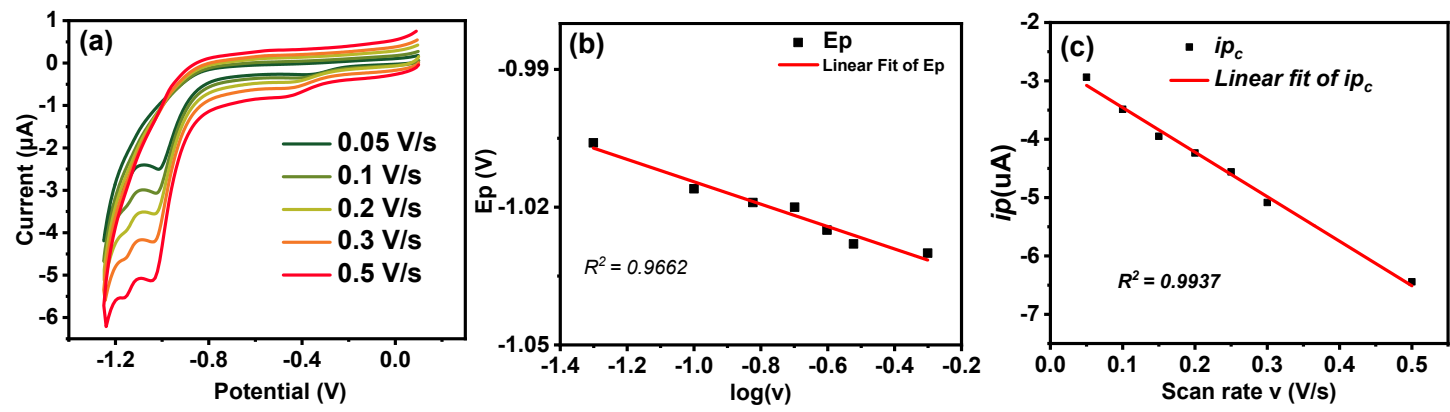

Figure S4. (a) Cyclic voltammograms recorded for the reduction of $8 \mathrm{BrAd}$ in PBS solution of $\mathrm{pH} 8$ at scan rates from $0.05 \mathrm{~V} / \mathrm{s}$ to $0.5 \mathrm{~V} / \mathrm{s}$. (b) Peak potential $\mathrm{E}_{p}$ and the linear fitting of different scan rates $(v)$. (c) Peak current $i_{p}$ and the linear fitting of scan rates $v$.

Table S3. Data for the Electrochemical Reduction of 8BrAd in pH 8 PBS solution.

\begin{tabular}{cccccc}
\hline Scan rate $v=0.1$ & $\boldsymbol{E}_{\boldsymbol{p}}{ }^{\mathbf{a}}$ & $-\partial E_{p} / \partial \log v$ & $\boldsymbol{\alpha}^{\mathbf{b}}$ & $E_{\frac{p}{2}}-E_{P}$ & $\boldsymbol{\alpha}^{\mathbf{c}}$ \\
\hline 8BrAd & -1.01 & 0.0243 & 0.61 & 0.0622 & 0.677 \\
\hline
\end{tabular}


${ }^{a}$ Obtained at $v=0.1 \mathrm{~V} / \mathrm{s}, \mathrm{n}=2,{ }^{b}$ Calculated from $\alpha=-1.15 n R T / F\left(\partial E_{p} / \partial \log v\right)$

when the scanning rates were used in the range of $0.05-0.5 \mathrm{~V} / \mathrm{s}$. The values calculated from $\alpha=1.857 R T / F\left(E_{\frac{p}{2}}-E_{P}\right)$.

Cyclic voltammetry obtained at Ag pertaining to the first reduction process, which involves the $\mathrm{C}-\mathrm{Br}$ bond shown in Fig S1. The peak current, $i_{\mathrm{pc}}$, varies linearly with $v$ and $v^{1 / 2}$, which points out a reduction process under mixed adsorption and diffusion control. The results are summarized in Table S3. The average values of $\alpha$ calculated from both $-\partial E_{p} / \partial \log v$ and $E_{\frac{p}{2}}-E_{P}$ are greater than 0.5 , indication the occurrence of a stepwise mechanism.

\section{EC-SERS of 8BrAd in different pH values.}
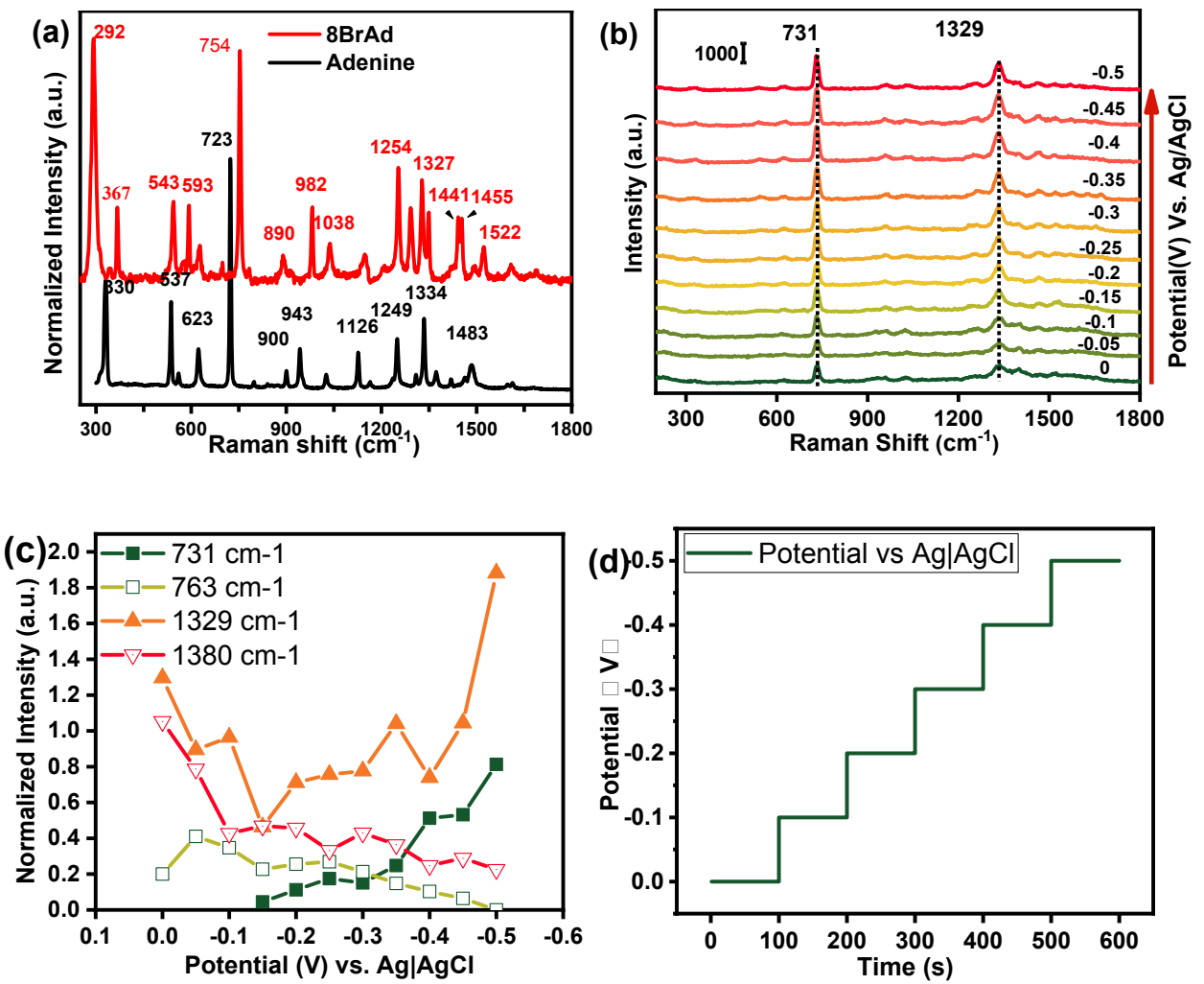

Figure S5. (a) Solid Raman spectra of Ad (black) and 8BrAd (red), respectively. (b) ECSERS of adsorbed Adenine on Ag NPs@Ag electrode with laser 532 nm, 0.1 mW. The potential interval is $0.05 \mathrm{~V}$. (c) Dependence of SERS integrated intensity of band at 731 $\mathrm{cm}^{-1}$, $, 763 \mathrm{~cm}^{-1}, \mathrm{\square}, 1329 \mathrm{~cm}^{-1}, \boldsymbol{\Delta}, 1380 \mathrm{~cm}^{-1}, \triangle$, in different applied potentials, normalized by adenine SERS spectra intensity in the same condition to remove the effect of chemical enhancement. (d) The potential step of electro-reflectance spectra and EC- 
SERS spectra were collected when the current keeps constant in a long time after the potential step.
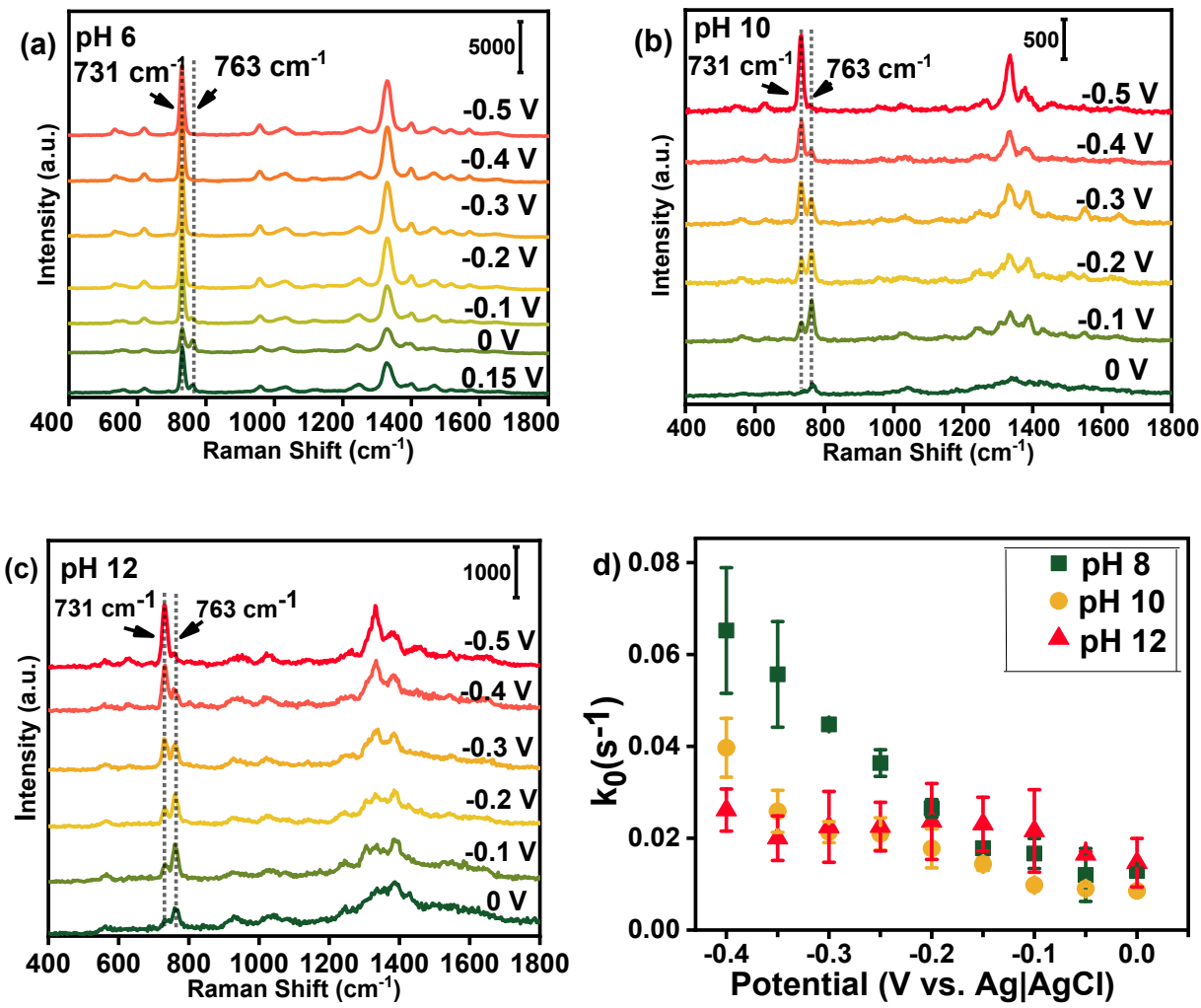

Figure S6. EC-SERS spectra of the 8BrAd adsorbed on silver nanoparticles modified silver electrode in different PBS solutions, $\mathrm{pH}$ 6(a), pH 10(b), and pH 12(c), respectively. In PBS pH 6 electrolyte solution, adenine showed up in relatively positive potentials in same acquisition time. While in the alkaline PBS solution, the adenine shows up in the potential negative than $-0.15 \mathrm{~V}$. The change of these EC-SERS spectra was similar in different $\mathrm{pH}$ values. All the spectra are detected with $532 \mathrm{~nm}$. (d) Apparent rate constants $\mathrm{k}_{0}$ at different $\mathrm{pH}$ values were plotted against applied potentials with laser line $532 \mathrm{~nm}$ and a laser power $0.05 \mathrm{~mW}$.

The $\boldsymbol{k}_{\boldsymbol{0}}$ increases almost linearly in $\mathrm{pH} 8$ when applied potentials moved negatively. While in the $\mathrm{pH} 10$ and 12 , the $\boldsymbol{k}_{\boldsymbol{0}}$ slowly increases when applied potential was more positive than $-0.35 \mathrm{~V}$, and then increases rapidly at the potential more negative than $0.35 \mathrm{~V}$. This can be explained that the high $\mathrm{pH}$ value affects the surface structure due to the hydroxide $\mathrm{OH}^{-}$anion strongly adsorbed on silver. 


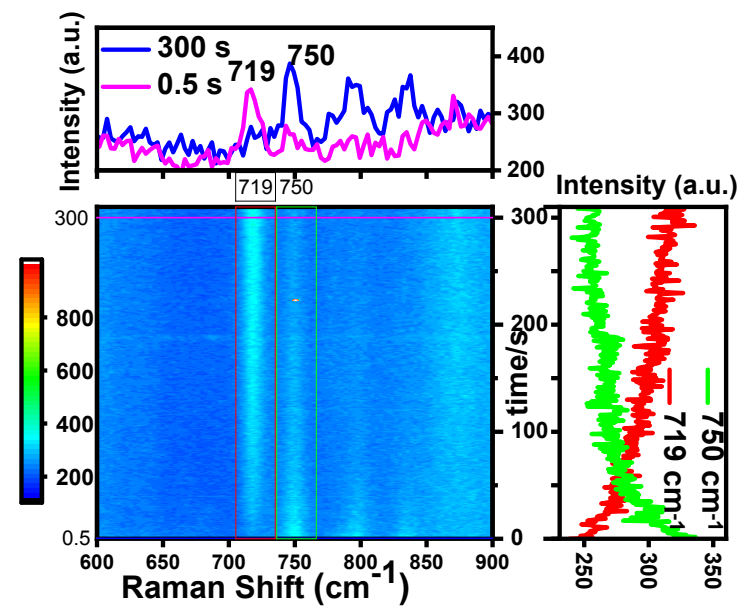

Figure S7. EC-SERS spectral contour map of 8BrAd in PBS (pH 8) deuterium aqueous solution.

\section{CV curves of photoelectrochemical cell}

As depicted in Figure S8, CV curves are detected under illuminating different wavelength with a low scan rate $20 \mathrm{mV} / \mathrm{s}$, with a defocusing mode of the light spot on the electrode surface $(\mathrm{d}=2 \mathrm{~mm})$. CV curves with laser $532 \mathrm{~nm}$ exhibits a slightly higher peak current than 633 and $785 \mathrm{~nm}$, while the on-set potential has not changed much. There is a little peak appeared at $-0.3 \mathrm{~V}$, in addition to be assumed as the $8 \mathrm{BrAd}$ reduction under the illumination.

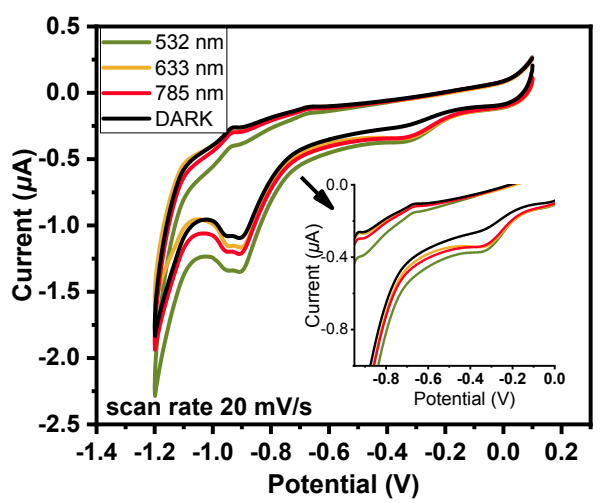

Figure S8. CV curves of photoelectrochemical cell under the different laser wavelength $532,633,785 \mathrm{~nm}$. All the laser power are $2 \mathrm{~nm}$, with a defocusing the light spot on the electrode surface $(\mathrm{d}=2 \mathrm{~mm})$. Electrolyte is $1 \mathrm{mM} 8 \mathrm{BrAd}$ and in $\mathrm{pH} 8$ solution, and at scan rate of $20 \mathrm{mV} \mathrm{s}^{-1}$.

6. Calculation details of electron transfer rate constants and the energy of molecules. 
For $8 \mathrm{BrAd}$ adsorbed on silver surfaces, we adopted a molecule-metallic cluster model of $8 \mathrm{BrAd}-\mathrm{Ag}_{18}$ to mimick adorption and reaction processes. The theoretical calculations, including geometry optimization, the scan of potential energy surfaces of ground and excited states were performed by using Gaussian 09 software package. Density functional theory calculations were carried out with Becke's three-parameter hybrid exchange functional (B3) and Lee-Yang-Parr's nonlocal correlation functional (LYP) approach, B3LYP. ${ }^{4 a}$, b, c The basis sets for $\mathrm{C}, \mathrm{N}, \mathrm{Br}$, and $\mathrm{H}$ atoms of investigated molecules were $6-311+\mathrm{G}(\mathrm{d}, \mathrm{p})$, which included the polarization function to all four kinds of atoms and only diffuse functions to $\mathrm{C}, \mathrm{N}$, and $\mathrm{Br}$ atoms. ${ }^{5 \mathrm{a}, \mathrm{b}}$ For $\mathrm{Ag}$ atoms, the valence electrons and inner shells were described by the basis set of LANL2DZ, and the corresponding relativistic effective core potentials, respectively. ${ }^{6 a}$, b Solvent effect was considered by the integral equation formalism polarization continuum model (PCM). ${ }^{7}$ Water with a dielectric constant of $\varepsilon=78.3$ was chosen as the solvent. The climbingimage nudged elastic band (Cl-NEB) method was used to define the states of the cleavage process, between the optimized initial states and final states, as shown in Figure S9.

(a) The initial state of cleavage process:

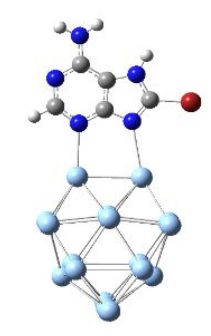

(b)The final state of cleavage process:

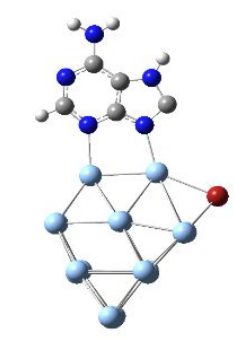

(c) The reaction path of cleavage process. 

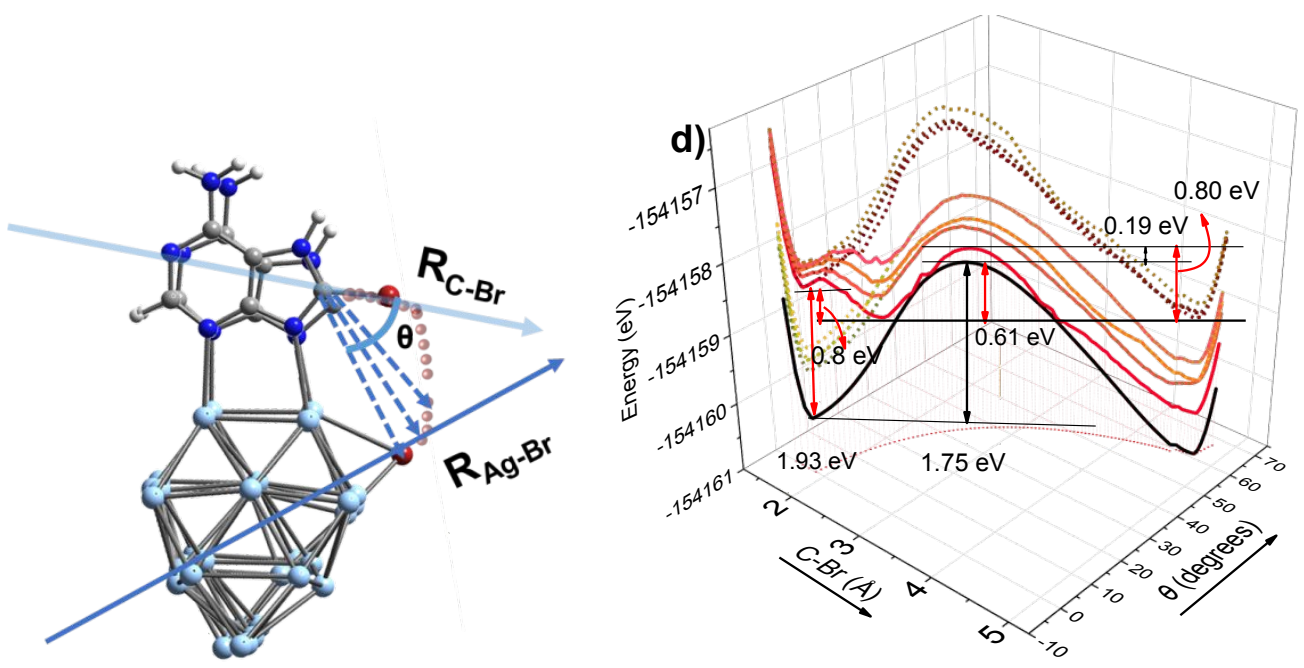

Figure S9. Optimized structures of (a) initial and (b) final states at corresponding potential energy minima. (c) The cleavage pathway of the $\mathrm{C}-\mathrm{Br}$ bond in the 8BrAd$\mathrm{Ag}_{18}$ cluster. (d) The relevant electronic states involved to the ground and excited states in the $\mathrm{C}-\mathrm{Br}$ bond breaking process without solvation.

\section{The derivation of $k_{1}$ by absorption coefficient $\alpha$.}

According to the Fermi golden rule, absorption rate constant $\boldsymbol{k}_{\mathbf{1}}$ can be expressed as:

$$
k_{1}=\frac{\pi}{2 \mathrm{~h}^{2}} \sum_{k} \sum_{m} P_{k}\left|\mu_{m k} \mathrm{~g} E_{0}\right|^{2} D\left(\omega_{m k}-\omega\right)
$$

And the total amount of light energy absorbed per unit time is given by,

$$
I=\frac{c}{8 \pi}\left|\stackrel{r}{E_{0}}\right|^{2}
$$

where $c$ denotes the light velocity. The obtained $\boldsymbol{k}_{\boldsymbol{l}}$,

$$
k_{1}=\frac{4 \pi^{2}}{3 h^{2} c} I \sum_{k} \sum_{m} P_{k}\left|\mu_{m k}\right|^{2} D\left(\omega_{m k}-\omega\right)
$$

According to the Beer-Lambert law the light intensity could be depicted as 


$$
A|d I|=N k_{1} h v
$$

The change of intensity is proportional to the light intensity $I$, the light path $d l$ and the light sensitizer concentration $C$ :

$$
d I=-\alpha(\omega) C I d l
$$

$N$ represents the number of $\mathrm{M}^{*}$ in the volume $\mathrm{Adl}$,

$$
N=C A d l N_{A}
$$

The absorption rate constant to generate hot electrons $\boldsymbol{k}_{1}$ :

$$
k_{1}=\frac{\alpha(\omega) I}{N_{A} h v}
$$

This is a simple derivation process of Eq. 11 in Text.

\section{Finite Element Method (FEM) simulation of absorption of plasmonic Ag electrode of nanostructure.}

(a)
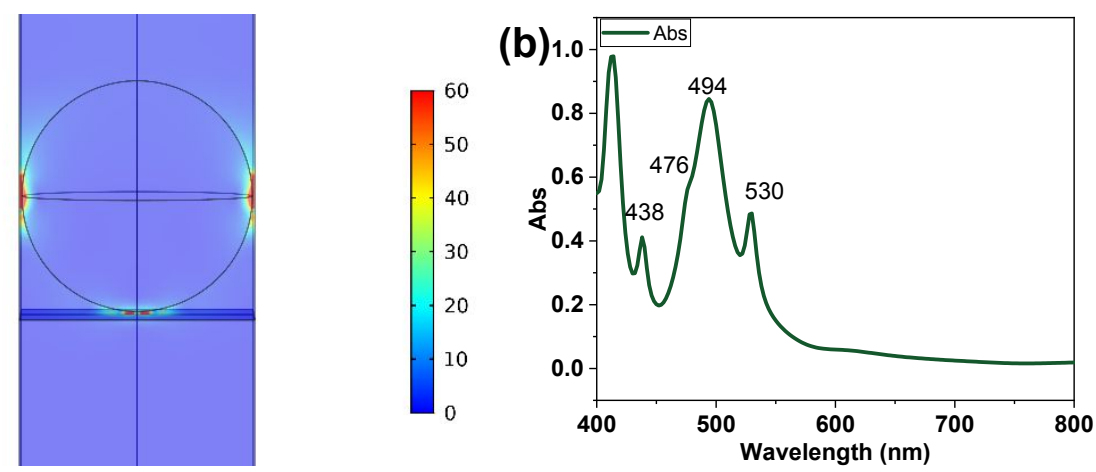

Figure S10. (a) Electromagnetic model of silver nanoparticles modified silver surfaces and (b) simulated absorption spectra of the electrode interfaces.

Finite Element Method (FEM) simulation was implemented to find the reflectance and absorbance using wave optical module of COMSOL Multiphysics. A three dimensional model (Figure S10a) was established representing 2D silver nanoparticle 
array on silver electrode in aqueous solution. Incident of light in normal direction (z) was implemented by port boundary condition, with linear polarization along y axis. Perfect electric conductor and perfect magnetic conductor boundary conditions were used to cut the model into one-fourth while keeping the periodicity ${ }^{8}$. Refractive index of aqueous solution was taken as 1.33 , and refractive index of silver was taken from experimental data ${ }^{9}$. The nanoparticle diameter set in the simulation was $70 \mathrm{~nm}$, and both interparticle distance and substrate-particle distance both at $1 \mathrm{~nm}$. The calculated absorbance electric field enhancement factor (local $\mathrm{E}$ field strength divided by incident E field strength) around particle at $492 \mathrm{~nm}$ as shown in Figure S10b.

\section{Reference}

(1) Lee, P. C. and Meisel, D., Adsorption and surface-enhanced Raman of dyes on silver and gold sols. The Journal of Physical Chemistry. 1982, 86, 3391.

(2) Xu, Y., Konrad, M. P., Trotter, J. L., McCoy, C. P. and Bell, S. E., Rapid One-Pot Preparation of Large Freestanding Nanoparticle-Polymer Films. Small. 2017, 13, 1602163.

(3) Suh, J. S., Jang, N. H., Jeong, D. H. and Moskovits, M., Adsorbate Photochemistry on a Colloid Surface: Phthalazine on Silver. The Journal of Physical Chemistry. 1996, 100, 805.

(4) (a) Lee, C., Yang, W. and Parr, R. G., Development of the Colle-Salvetti correlation-energy formula into a functional of the electron density. Physical review B. 1988, 37, 785; (b) Becke, A. D., Densityfunctional thermochemistry. III. The role of exact exchange. The Journal of Chemical Physics. 1993, 98, 5648; (c) Becke, A. D., Density-functional thermochemistry. IV. A new dynamical correlation functional and implications for exact-exchange mixing. The Journal of Chemical Physics. 1996, 104, 1040.

(5) (a) Krishnan, R., Binkley, J. S., Seeger, R. and Pople, J. A., Self-consistent molecular orbital methods. XX. A basis set for correlated wave functions. The Journal of Chemical Physics. 1980, 72, 650; (b) McLean, A. and Chandler, G., Contracted Gaussian basis sets for molecular calculations. I. Second row atoms, $Z=11-18$. The Journal of Chemical Physics. 1980, 72, 5639.

(6) (a) Hay, P. J. and Wadt, W. R., Ab initio effective core potentials for molecular calculations. Potentials for the transition metal atoms Sc to Hg. The Journal of Chemical Physics. 1985, 82, 270; (b) Hay, P. J. and Wadt, W. R., Ab initio effective core potentials for molecular calculations. Potentials for K to Au including the outermost core orbitals. The Journal of Chemical Physics. 1985, 82, 299.

(7) Foresman, J. B., Keith, T. A., Wiberg, K. B., Snoonian, J. and Frisch, M. J., Solvent Effects. 5. Influence of Cavity Shape, Truncation of Electrostatics, and Electron Correlation on ab Initio Reaction Field Calculations. The Journal of Physical Chemistry. 1996, 100, 16098.

(8) Hutter, T., Huang, F. M., Elliott, S. R. and Mahajan, S., Near-Field Plasmonics of an Individual Dielectric Nanoparticle above a Metallic Substrate. The Journal of Physical Chemistry C. 2013, 117, 7784.

(9) Johnson, P. B. and Christy, R. W., Optical Constants of the Noble Metals. Physical Review B. 1972, 
6, 4370 . 